\title{
Analysis and in situ characterization of activated sludge communities capable of benzotriazole biodegradation
}

\author{
Bastian Herzog ${ }^{1 *+}$, Bettina Huber ${ }^{1 \dagger}$, Hilde Lemmer ${ }^{2}$, Harald Horn $^{3}$ and Elisabeth Müller ${ }^{1}$
}

\begin{abstract}
Background: The aerobic biodegradation potential of benzotriazole (BTri), 4-, and 5-tolyltriazole (4-TTri, 5-Tri) by activated sludge communities (ASC), taken from three wastewater treatment plants (WWTP): a membrane bioreactor (MBR-MH), a conventional activated sludge plant (CAS-E) with intermittent nitrification/denitrification, and a CAS-plant (CAS-M) with a two-stage activated sludge treatment, is evaluated.

Results: In small-scale setups (500 mL), 5-TTri (31.2 mg/L) and BTri (34.0 mg/L) were eliminated under aerobic conditions. 5-TTri was removed after 7 days while the ASC showed significant differences concerning BTri biodegradation. ASC from MBR-MH removed BTri after 21 days while CAS-E and CAS-M needed 42 and 49 days, respectively, but after acclimation (49 days), all ASC biodegraded BTri within 7 days. In the larger-scale setups (10 L), after 4 days, 96\% 5-TTri (0.5 mg/L) was already removed while BTri $(1.0 \mathrm{mg} / \mathrm{L})$ was eliminated after 7 days. 4-TTri proved stable even after 105 days in both setups, regardless the concentration applied. Fluorescence in situ hybridization (FISH) analysis revealed the Alpha- and Betaproteobacteria as the dominant groups while the abundance of the Actinobacteria significantly decreased.

Conclusions: ASC biodegraded BTri and 5-TTri under aerobic, mesophilic conditions in all setups. Biodegradation was fastest for 5-Tri, while BTri biodegradation was affected by concentration: BTri was readily biodegraded at low concentrations while biodegradation at high concentrations was significantly slower and 4-Tri was not removed at all. Additional experiments, using the FISH technique to analyze the ASC structure, showed that the two groups Alpha- and Betaproteobacteria most likely are involved in the biodegradation of BTri and 5-TTri.
\end{abstract}

Keywords: Tolyltriazole; 1-H-benzotriazole; 4-methyl-benzotriazole; 5-methyl-benzotriazole; Xenobiotics; FISH

\section{Background}

The xenobiotic compounds benzotriazole (BTri), 5tolyltriazole (5-TTri), and 4-tolyltriazole (4-TTri) are polar micropollutants commonly used as corrosion inhibitors. 4- and 5-TTri are commercially available as tolyltriazole mixture (TTri) that is widely used in metal finishing and as corrosion protection in cooling systems $[1,2]$. Among other applications, these three benzotriazole species are mainly used as anticorrosives in aircraft deicing or breaking fluids [3], in household dishwashing detergents for silver protection [4], and as UV stabilizers

\footnotetext{
* Correspondence: b.herzog@tum.de

${ }^{\dagger}$ Equal contributors

'Urban Water Systems Engineering, Technische Universität München, Am Coulombwall, Garching 85748, Germany

Full list of author information is available at the end of the article
}

[5,6]. Due to an annual production volume of around 9,000 tons in the USA and probably a much higher global production $[7,8]$, their widespread usage, high polarity (logP 1.26 and 1.78 for BTri and TTri, respectively), high water solubility, and poor biodegradability, these compounds occur in nearly all aquatic compartments [9-12]. This includes ground water with up to $1,548 \mathrm{ng} / \mathrm{L}$ (all three compounds) [13], river systems with up to 7,997 ng/L for BTri, and 19,396 ng/L for TTri [14], and the North Sea (up to $40 \mathrm{ng} / \mathrm{L}$, all three benzotriazoles) [15,16]. Highest concentrations of 128 and $198 \mathrm{mg} / \mathrm{L}$ for BTri and TTri, respectively, were detected at a perched water monitoring well at an international airport [17]. Concentrations above $40 \mathrm{mg} / \mathrm{L}$ for BTri and $6 \mathrm{mg} / \mathrm{L}$ for 5-TTri show toxic effects in Microtox tests (Vibrio fischeri light emission as toxicity test system, Azur 
Environmental, Oceanside, CA, USA) and other organisms [18-20]. Thus, benzotriazoles might exhibit detrimental effects on aquatic systems [21]. However, due to their high efficiency in protecting metal surfaces from corrosion, their low costs, and a general low toxicity, these chemicals are frequently used in industrial and household applications [7,22]. As wastewater treatment plants (WWTP) are incapable of completely removing these compounds during the treatment process [6,23-25], WWTP effluents constitute a major point source for benzotriazoles in the receiving rivers. Several workgroups reported different removal efficiencies in conventional WWTP ranging from $13 \%$ to $62 \%$ for BTri, $11 \%$ to $74 \%$ for 5 -TTri, and far less for 4-TTri [26-29]. At laboratory conditions, biological removal of BTri is also incomplete while one study with 5TTri showed its biodegradation under aerobic conditions within 91 days [9]. Reported half-lives of BTri and 5-TTri with 114 and 14 days [9], respectively, are not applicable in WWTP where hydraulic retention times (HRT) are often below $15 \mathrm{~h}$ during biological treatment [9]. Therefore, these three compounds are ubiquitous in river water across Europe receiving WWTP effluents [14].

This study aimed to evaluate the biodegradation capacity of activated sludge communities (ASC) regarding three benzotriazoles. Three ASC were taken from three WWTP with different treatment regimes, i.e., a membrane bioreactor (MBR-MH), and two conventional activated sludge (CAS) treatment plants, and tested for their ability to aerobically biodegrade different benzotriazoles at different concentrations. In addition, larger-scale setups (10 L), supplied with low concentrations $(\leq 1.0 \mathrm{mg} / \mathrm{L})$ of benzotriazoles, were performed to evaluate the degree of biodegradation with respect to benzotriazole concentration. Furthermore, fluorescence in situ hybridization (FISH) was applied to characterize the ASC structure and to identify the bacterial groups most likely contributing to benzotriazole biodegradation.

\section{Results and discussion}

Biodegradation of BTri, 4-TTri, and 5-TTri under aerobic conditions

\section{Small-scale setups}

All controls showed that the benzotriazoles BTri, 4TTri, and 5-TTri were stable under sterile and abiotic conditions, showing that removal in the experimental setups was due to biodegradation. All three ASC were able to biodegrade BTri under aerobic conditions. However, differences in biodegradation pattern, biodegradation rate $\left(\mathrm{mg} / \mathrm{L}\right.$ day $\left.^{-1}\right)$, and incubation time were observed. Different organisms and/or differences in the ASC composition might affect biodegradation of BTri (see Table 1). All ASC started with a lag phase of 7 days. BTri was removed in 21 days in ASC from MBR-MH, while the other two ASC needed 42 and 49 days for CAS-E and CAS-M, respectively. Biodegradation in the ASC did not follow a mathematical model, as proposed by [9], and thus is difficult to describe. Assuming that biodegradation would follow an almost linear removal, biodegradation rates of $0.52 \mathrm{mg} / \mathrm{L} \mathrm{day}{ }^{-1}$ for CAS-E, $0.70 \mathrm{mg} / \mathrm{L} \mathrm{day}^{-1}$ for CAS-M, and $1.00 \mathrm{mg} / \mathrm{L} \mathrm{day}^{-1}$ for MBR-MH were achieved. Such different rates might be due to the composition of the sludge communities. It might also be that sludge age affected biodegradation as it was shown that this is a crucial factor for the biodegradation of problematic substances [29].

In addition, an acclimation effect was observed for BTri, leading to a rapid elimination after acclimation (49 days) of the ASC. To elucidate this effect, $10 \mathrm{mg} / \mathrm{L}$ BTri were supplied to the same sludge again that already eliminated BTri. Due to acclimation, all ASC from MBR-MH, CAS-E, and CAS-M eliminated BTri in 7 days. The organisms, responsible for the biodegradation, might require a certain time to adapt to high BTri concentrations. This result shows that once adapted, the ASC might be able to degrade even higher BTri concentrations than the ones applied here. The removal capacity of ASC might be enhanced for BTri biodegradation by means of acclimation. BTri, present at $\times 1,000$ lower (i.e., $\mu \mathrm{g} / \mathrm{L}$ ) concentrations in WWTP could be efficiently removed by such an acclimated ASC.

In contrast to BTri, biodegradation of 4-TTri and 5TTri showed a completely different behavior. 5-TTri was biodegraded in all tested ASC in around 7 days to a concentration below the detection limit, not requiring acclimation, while 4-TTri was refractory (Figure 1). After 49 days, 4-TTri was still present at the initial concentration, regardless the ASC. This result is in agreement with literature data where 4-TTri was reported to be

Table 1 Sludge characteristics of the different activated sludge communities tested

\begin{tabular}{|c|c|c|c|c|}
\hline WWTP & Sludge composition & Color & Settleability & $S V I^{a}$ \\
\hline MBR-MH & $\begin{array}{c}\text { Well-suspended biomass with many planktonic organisms, } \\
\text { homogenous suspension, supernatant turbid }\end{array}$ & Black to dark-brown & Slow & 88 \\
\hline CAS-M & $\begin{array}{l}\text { Few planktonic organisms, inhomogeneous } \\
\text { suspension, supernatant clear }\end{array}$ & Gray to light gray & Fast & 86 \\
\hline CAS-E & $\begin{array}{c}\text { Numerous planktonic organisms, suspension somewhat } \\
\text { homogenous, supernatant turbid }\end{array}$ & Anthracite & Slow to moderate & 88 \\
\hline
\end{tabular}

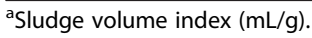




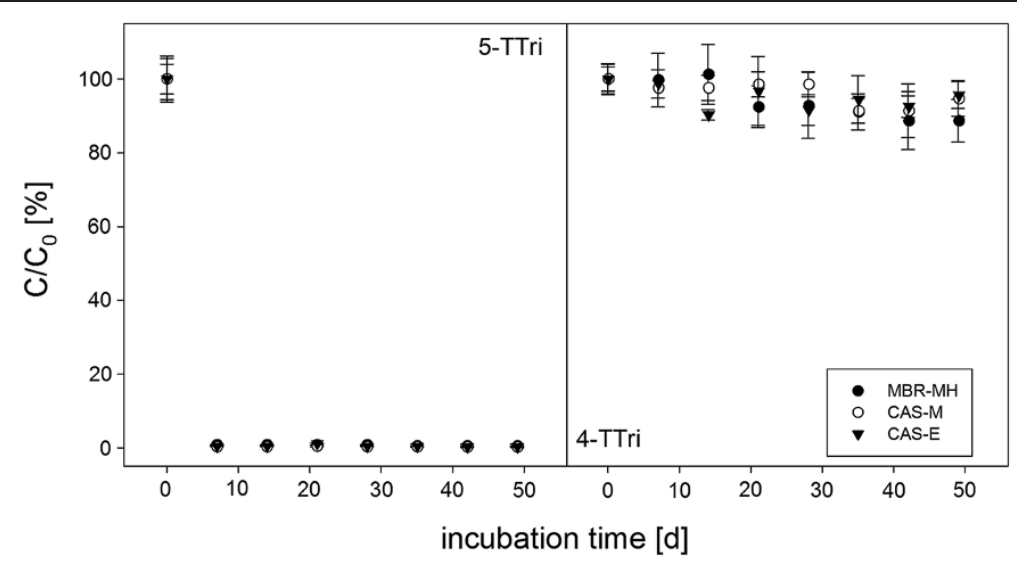

Figure 1 Biodegradation behavior of aerobic 4- and 5-TTri. Aerobic 5-TTri biodegradation behavior in the three ASC (initial concentration is given in Table 2). Shown are mean values of 5-TTri concentrations of duplicate experiments with error bars indicating standard deviations $(n=2)$. Aerobic 4-TTri biodegradation behavior in the three ASC (initial concentration is given in Table 2). Shown are mean values of 4-TTri concentrations of duplicate experiments. MBR, membrane bioreactor; CAS, conventional activated sludge treatment.

quite stable during wastewater treatment and characterized by poor removal $[8,26,30]$. Regarding 5 -TTri, acclimation as observed for BTri was not found as it was already degraded in the first week. Additionally, transformation of 5-TTri into either BTri or 4-TTri, as suggested by [9], was not found in the ASC. As 5-TTri was already degraded in 7 days, biodegradation rates were assumed with $4.50 \mathrm{mg} / \mathrm{L} \mathrm{day}^{-1}$ (CAS-M), $3.26 \mathrm{mg} / \mathrm{L} \mathrm{day}^{-1}$ (CAS-E), and $1.90 \mathrm{mg} / \mathrm{L} \mathrm{day}^{-1}$ (MBR-MH). Biodegradation rates might thus only depend on the amount of 5-TTri initially present. It seems likely that the biodegradation capacity of the sludge communities was not fully reached. After supplying TTri again, a similar behavior was observed: 5-TTri was biodegraded in 7 days while 4-TTri remained unchanged (Table 2).

It might be that the shift of one methyl group to the next adjacent carbon atom makes 5-TTri, in contrast to 4-TTri, a readily biodegradable substance. This effect fits the reported behavior in WWTP [9,27]. Why the position of the methyl group has such a dramatic influence on biodegradation is still unknown and topic to further research. Steric hindrance of the enzyme(s) catalyzing degradation of 4-TTri might play a role. Enzymes are known for discriminating substrates due to different substitution patterns [31-34]. As 4- and 5-TTri are constitutional isomers, it seems likely that the position of the methyl group effects enzymatic biodegradability.

A third supply of $10 \mathrm{mg} / \mathrm{L}$ TTri and BTri at day 77 was performed to test whether 4-TTri might require a longer incubation to be removed. In this batch phase, no sampling or peptone addition was performed. Such conditions created a nutrient shortage as BTri, 5-TTri, and peptone were presumably consumed within the first 7 days. As the only remaining nutrient source was 4TTri, the organisms should be forced to metabolize this compound in order to avoid starvation. However, even after 105 days of incubation, 4-TTri was not removed (Figure 2). As already shown, BTri and 5-TTri were

Table 2 Activated sludge inocula and dosage regime for BTri, 4-TTri, and 5-TTri in the two biodegradation experiments (small-scale and larger-scale setups)

\begin{tabular}{|c|c|c|c|c|c|c|c|c|}
\hline & \multirow[t]{2}{*}{ Origin of inoculum } & \multicolumn{3}{|c|}{$\begin{array}{l}\text { Initial concentration } \\
(\mathrm{mg} / \mathrm{L})\end{array}$} & \multicolumn{2}{|c|}{$\begin{array}{l}\text { Single dosage after } \\
49 \text { days }(\mathrm{mg} / \mathrm{L})\end{array}$} & \multicolumn{2}{|c|}{$\begin{array}{l}\text { Single dosage after } 77 \text { days } \\
\text { (batch-test) (mg/L) }\end{array}$} \\
\hline & & BTri & 4-TTri & 5-TTri & BTri & TTri & BTri & TTri \\
\hline \multirow[t]{6}{*}{ Small-scale setup } & MBR-MH & 21.0 & 8.8 & 13.2 & 10.0 & 10.0 & 10.0 & 10.0 \\
\hline & CAS-M & 34.0 & 20.8 & 31.2 & 10.0 & 10.0 & 10.0 & 10.0 \\
\hline & CAS-E & 22.0 & 15.2 & 22.8 & 10.0 & 10.0 & 10.0 & 10.0 \\
\hline & Experimental phase & & । & & \multicolumn{2}{|c|}{$\|$} & \multicolumn{2}{|c|}{ III } \\
\hline & Duration (day) & & 49 & & \multicolumn{2}{|c|}{28} & \multicolumn{2}{|c|}{28} \\
\hline & Total duration of the experiment (day) & & 49 & & \multicolumn{2}{|c|}{77} & \multicolumn{2}{|c|}{105} \\
\hline \multirow[t]{2}{*}{ Larger-scale setup } & CAS-M & 1.03 & 0.38 & 0.53 & \multicolumn{2}{|c|}{-} & \multicolumn{2}{|c|}{-} \\
\hline & Total duration of the experiment (day) & \multicolumn{3}{|c|}{22} & \multicolumn{2}{|c|}{-} & \multicolumn{2}{|c|}{ - } \\
\hline
\end{tabular}

Tri consists of 4- and 5-Tri 40\%/60\% (w/w). 


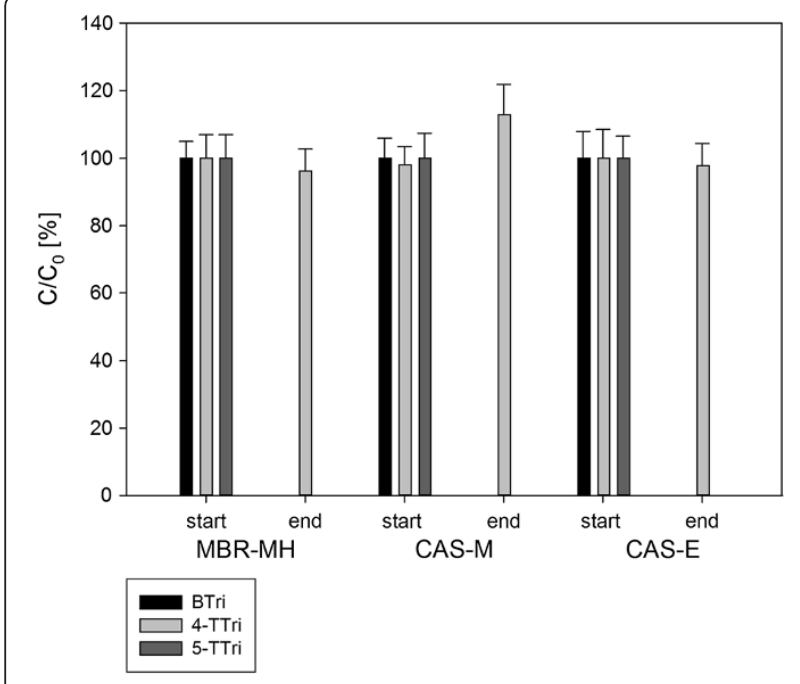

Figure 2 Aerobic biodegradation during a 4-week batch experiment without sampling. Benzotriazole concentrations were determined at the start and the end of the experiment. Shown are mean values of benzotriazole concentrations of duplicate experiments with error bars indicating standard deviations $(n=2)$.

completely removed again. These findings confirm the following biodegradation order: 5-TTri, BTri, and 4-TTri that was refractory during the experiment.

\section{Larger-scale setups}

To evaluate the biodegradation behavior of the ASC in the presence of lower and thus environmentally more relevant benzotriazole concentrations of $1 \mathrm{mg} / \mathrm{L}$ BTri and TTri respectively, further experiments were conducted with larger-scale setups $(10 \mathrm{~L})$. As all small-scale setups biodegraded BTri and 5-TTri, for the following experiments, only ASC from CAS-M was used.

All three benzotriazoles, i.e., BTri, 4-TTri, and 5-TTri, were stable under sterile and abiotic conditions. Neither sorption to activated sludge nor volatilization occurred, thus the benzotriazoles were removed by biodegradation. The benzotriazole measurements of the larger-scale setups showed that BTri and 5-TTri were removed by the aerobic ASC within 7 days of incubation (Figure 3). This is significantly faster compared to the small-scale setups were BTri was removed within 49 days without acclimation in ASC from CAS-M. This slow removal in the small-scale setups might be attributed to possible toxic effects due to the high initial concentration of $34 \mathrm{mg} / \mathrm{L}$ (Table 2), as concentrations above $40 \mathrm{mg} / \mathrm{L}$ were already shown to be detrimental [18].

In contrast to BTri, the biodegradation of 5-TTri was faster, since after 4 days more than $96 \%$ were already removed and 5-TTri might presumably be degraded on day 5 . As in the small-scale setups, the first measurement was performed on day 7 were already 5-TTri was

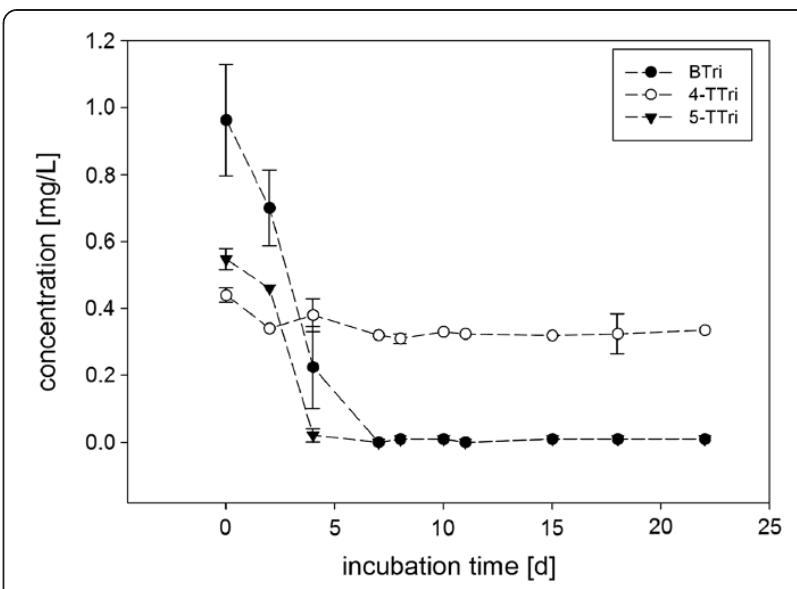

Figure 3 Aerobic biodegradation of BTri, 4-, and 5-TTri in the larger-scale setups during 18 days of incubation. Initial benzotriazoles concentrations are given in Table 2. Shown are mean values of benzotriazole concentrations of duplicate experiments with error bars indicating standard deviations $(n=2)$.

degraded, more measurements were performed during the larger-scale setups to evaluate the 5-TTri biodegradation pattern: after a short lag phase (day 0 to day 2 ) almost all 5-TTri was removed within the following 2 days. Therefore, 5-TTri biodegradation in the ASC was seemingly not affected by the supplied concentration as the removal in the small-scale setups was comparably as fast as in the larger-scale setups although the concentration was around 60 times higher. No effects attributed to toxicity, as observed with BTri, occurred whereas concentrations above $6 \mathrm{mg} / \mathrm{L}$ already showed toxic effects for Vibrio fisheri [18].

4-TTri, as already observed in the small-scale setups, was refractory during the experiment. This is in accordance with literature data where 4-TTri was also found to be almost stable during wastewater treatment $[8,26,30]$. Thus, the degradation behavior of 4-TTri might not depend on the used concentrations (Table 2) but more likely on the molecular structure of the compound itself.

\section{Assessing the ASC structure by fluorescence in situ hybridization}

FISH with group-specific rRNA-targeted oligonucleotide probes was applied to characterize the composition of the ASC in the inoculum and setups 1 to 4 without prior isolation and/or cultivation. The aim was to gain information about diversity and consistence of the ASC and to identify microorganisms important for benzotriazole biodegradation. For this purpose, six different specific probes were used (Table 3) targeting the dominant bacterial groups important for wastewater treatment [35-37]. The signal intensities obtained with all rRNAtargeted oligonucleotide probes were strong indicating high cellular rRNA contents. 
Table 3 Group-specific rRNA-targeted oligonucleotide probes

\begin{tabular}{|c|c|c|c|c|c|}
\hline Probe & Target molecule & Specificity & $F A^{a}(\%)$ & Probe sequence $\left(5^{\prime}-3^{\prime}\right)$ & Reference \\
\hline BET42a & $23 \mathrm{~S}$ rRNA & Betaproteobacteria & 35 & GCCTTCCCACTTCGTTT & [38] \\
\hline GAM42a & $23 \mathrm{~S}$ rRNA & Gammaproteobacteria & 35 & GCCTTCCCACATCGTTT & [38] \\
\hline ALF968 & $16 \mathrm{~S}$ rRNA & Alphaproteobacteria & 20 & GGTAAGGTTCTGCGCGTT & [39] \\
\hline CF319a & $16 \mathrm{~S}$ rRNA & Most Flavobacteria, some Bacteroidetes, some Sphingobacteria & 35 & TGGTCCGTGTCTCAGTAC & [40] \\
\hline $\mathrm{LGCA} / \mathrm{B} / \mathrm{C}$ & $16 \mathrm{~S}$ rRNA & Firmicutes & 35 & TGGAAGATTCCCTACTGC & [41] \\
\hline HGC1156 & $16 \mathrm{~S}$ rRNA & Actinobacteria & 20 & CGAGTTGACCCCGGCACT & [42] \\
\hline
\end{tabular}

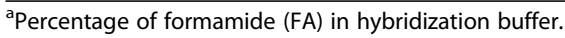

FISH analysis of the inoculum revealed that the Proteobacteria and Actinobacteria were the most frequently detected bacterial groups occurring in categories between 3 and 5 (Figure 4). In the group of Proteobacteria, the Betaproteobacteria were the most dominant ones in the activated sludge (category 5), followed by the Alphaproteobacteria (category 4), and, to a lesser extent, by the Gammaproteobacteria (category 3). The groups Firmicutes and Bacteroidetes played only a minor role in the ASC. These findings are in accordance with previous investigations $[43,44]$ where the alpha and gamma subclasses of Proteobacteria were only detected in low amounts in CAS-M [44].

At the end of the experiment, where all four setups were analyzed by FISH, the Betaproteobacteria dominated the activated sludge closely followed by the Alphaproteobacteria. In contrast to the inoculum, the amount of Gammaproteobacteria was far less and also the abundance of the Actinobacteria significantly decreased. Thus, three major

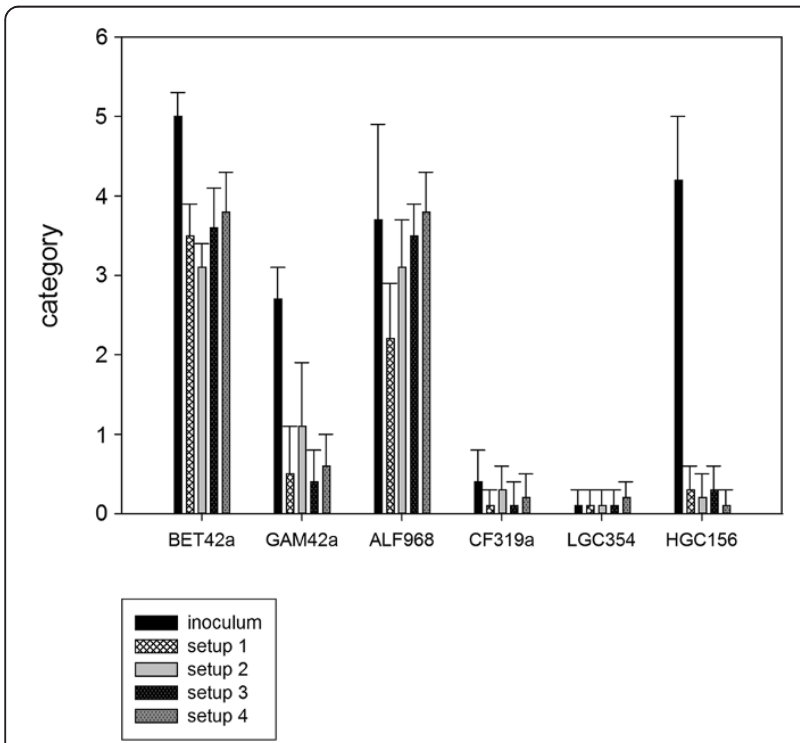

Figure 4 Quantitative FISH analysis of the larger-scale setups 1 to 4 and the inoculum. Error bars indicate standard deviations of the evaluation $(n=15)$. Setups 1 and 2: control setups without benzotriazoles; Setups 2 and 3: experimental setups supplied with benzotriazoles. shifts within the ASC were observed during incubation. The abundance of Actinobacteria dropped by almost four category units compared to the inoculum while the abundance of the Gammaproteobacteria significantly decreased from category 3 to below 1 . Finally, also the dominant Betaproteobacteria decreased from category 5 to below 4 .

As all three shifts in the ASC occurred within the negative controls (setups 1 and 2) and the benzotriazole supplied setups 3 and 4, they might be associated to the applied conditions rather than benzotriazole addition. The supplied specific nutrients $(100 \mathrm{mg} / \mathrm{L}$ carbon and $30 \mathrm{mg} / \mathrm{L}$ nitrogen) might not be sufficient for a large variety of microorganisms, especially for the Actinobacteria, were the applied conditions were detrimental as observed in significantly reduced abundance.

Generally, the abundance was highest in the inoculum but also the Alphaproteobacteria remained almost stable concerning the abundance of bacteria. In this group, the bacterial abundance in the benzotriazole supplied setups was even higher compared to the negative control setups, indicating that members of this group might contribute to benzotriazole biodegradation. Also, the group Betaproteobacteria might be associated with benzotriazole biodegradation as this group was, even at the end of the experiment, still the dominant one.

\section{Conclusions}

Activated sludge communities (ASC) collected from three wastewater treatment plants with different treatment regimes, were capable of eliminating BTri and 5TTri under aerobic, mesophilic conditions in small-scale and larger-scale setups. Biodegradation was fastest for 5TTri, regardless the supplied concentration ranging from 0.53 to $31.20 \mathrm{mg} / \mathrm{L}$, followed by BTri, that was well removed at a concentration of $1.03 \mathrm{mg} / \mathrm{L}$ while possible toxic effects negatively influenced its biodegradation at high concentrations. 4-TTri was refractory throughout the experiment and was not removed even after 105 days of incubation, regardless the supplied concentration. Additional experiments, using FISH technique to analyze the ASC structure, showed that the two groups Alphaand Betaproteobacteria most likely are involved in the biodegradation of BTri and 5-TTri. 


\section{Methods}

\section{Chemicals}

1-H-benzotriazole (BTri; CAS 95-14-7), 4-tolyltriazole (4-TTri; CAS 29878-31-7), and 5-tolyltriazole (5-TTri; CAS 136-85-6), known as tolyltriazole (TTri; CAS 29385-43-1), were provided by Cimachem $\mathrm{GmbH}$ (Kirchheimbolanden, Germany). The internal standard 5,6-dimethyl-benzotriazole used for GC-MS/MS, sodium carbonate solution, and toluol were purchased from Sigma-Aldrich (Steinheim, Germany). All other media components were obtained from Merck KGaA (Darmstadt, Germany). High-purity water was prepared by a Milli-Q system (Millipore, Billerica, MA, USA).

\section{Activated sludge sampling}

ASC samples (500 mL, collected in glass bottles) for inoculation were taken from the aeration tanks of the respective WWTP as described elsewhere [45]: From MBR-MH, a WWTP using membrane filtration, from CAS-M with two-stage activated sludge treatment, and from CAS-E, a conventional activated sludge (CAS) plant with intermittent nitrification/denitrification. All AS samples were taken as mixed liquor grab samples during aeration to ensure optimal mixing of the sludge. For inoculation of the laboratory reactors, the ASC were centrifuged (10 $\mathrm{min}, 4,000 \times g)$, the supernatants discarded, and the remaining biomass was washed with $1 \times$ PBS buffer $\left(\mathrm{NaCl} 8.0 \mathrm{~g} / \mathrm{L}, \mathrm{KCl} 0.2 \mathrm{~g} / \mathrm{L}, \mathrm{Na}_{2} \mathrm{HPO}_{4} 2.7 \mathrm{~g} / \mathrm{L}\right.$, $\mathrm{KH}_{2} \mathrm{PO}_{4} 0.2 \mathrm{~g} / \mathrm{L}$ ). The procedure was repeated twice to remove wastewater residues and nutrients.

\section{Experimental setups}

Two separate setups were performed: (A) Small-scale setups $(500 \mathrm{~mL})$ and $(B)$ larger-scale setups $(10 \mathrm{~L})$. To evaluate the biodegradation potentials of the three activated sludge communities, separate setups were performed.

Small-scale setups: To ensure high activity and proper growth of the organisms and to mimic wastewater nutrient conditions, $70 \mathrm{mg} / \mathrm{L}$ peptone was supplied at the start of the experiment and after each sampling. Setups were performed in 500-mL glass Erlenmeyer flasks with air-permeable aluminum caps filled with $150 \mathrm{~mL}$ of the following sterile media: a modified mineral salt media (adapted from DIN ISO 9888) consisting of $\mathrm{KH}_{2} \mathrm{PO}_{4}$ $0.08 \mathrm{~g} / \mathrm{L}, \quad \mathrm{K}_{2} \mathrm{HPO}_{4} \quad 0.20 \quad \mathrm{~g} / \mathrm{L}, \quad \mathrm{Na}_{2} \mathrm{HPO}_{4} \quad 0.30 \mathrm{~g} / \mathrm{L}$, $\mathrm{MgSO}_{4} * 7 \mathrm{H}_{2} \mathrm{O} 0.02 \mathrm{~g} / \mathrm{L}, \mathrm{CaCl} * 2 \mathrm{H}_{2} \mathrm{O} 0.04 \mathrm{~g} / \mathrm{L}, \mathrm{FeCl}_{3} * 6$ $\mathrm{H}_{2} \mathrm{O} 0.30 \mathrm{mg} / \mathrm{L}$ at $\mathrm{pH} 7.4$.

Larger-scale setups: Inoculation was performed with ASC from WWTP CAS-M only. BTri, 4-TTri, and 5TTri were analyzed separately to investigate different biodegradation patterns. To test the influence of specific nutrients on biodegradation of benzotriazoles, the following nutrients were supplied at the beginning of the experiment and after each sampling: sodium acetate*3 $\mathrm{H}_{2} \mathrm{O}(0.567 \mathrm{~g} / \mathrm{L}), \mathrm{NH}_{4} \mathrm{NO}_{3}(0.086 \mathrm{~g} / \mathrm{L})$, and $\mathrm{NH}_{4} \mathrm{Cl}$ $(0.057 \mathrm{~g} / \mathrm{L})$. Larger-scale setups were prepared in the same way as the small ones in 10-L plastic bottles (PE).

All media were spiked with BTri and TTri at varying concentrations according to Table 2 .

All setups were run in duplicate and inoculated with the ASC of one of the three WWTP (small-scale setups) and with ASC from CAS-M (larger-scale setups) to 4 and $2 \mathrm{~g} / \mathrm{L}$ mixed liquor suspended solids (MLSS), respectively.

Control setups, fed alike the experimental ones, were performed for (A) abiotic control without ASC and (B) sterile control with two-times autoclaved ASC. These controls were used to investigate abiotic effects. Aerobic conditions were ensured using an orbital shaker at $120 \mathrm{rpm}$ (small-scale setups) while larger-scale setups were continuously stirred. All experiments were carried out in the dark at $20^{\circ} \mathrm{C}\left( \pm 2^{\circ} \mathrm{C}\right)$. This temperature was sufficient for the activity of the organisms as the temperature was $14^{\circ} \mathrm{C}$ when the ASC were collected. The $\mathrm{pH}$ was controlled around 7 .

\section{Dosage regime and sampling}

Small-scale setups The experiment lasted for 105 days. Firstly, different amounts of benzotriazoles were supplied depending on the used sludge (Table 2). After BTri and 5-TTri were degraded, another $20 \mathrm{mg} / \mathrm{L} \mathrm{BTri/TTri}$ were supplied. Ten-milliliter samples were taken once a week from the setups after a 1-h sedimentation period to reduce the amount of biomass withdrawn with the supernatant. Samples were prepared as described in 'sample preparation' section.

Larger-scale setups Experiment was carried out over a 22-day period. The experimental setups (setups 3 and 4, Figure 3) were spiked with a maximum of $1 \mathrm{mg} / \mathrm{L} \mathrm{BTri}$ and TTri, respectively (exact values in Table 3 ) and sampled twice a week by withdrawing $60 \mathrm{~mL}$ from the setups as described above. Setups 1 and 2 (Figure 3) served as controls (no benzotriazole supply) to evaluate whether the benzotriazoles affected the ASC.

\section{Sample preparation and chemical analyses}

Samples obtained from the setups were centrifuged $\left(10 \mathrm{~min}, 8,000 \times g, 20^{\circ} \mathrm{C}\right)$ to remove cellular debris and biomass, filled into glass bottles, and stored in the dark at $-20^{\circ} \mathrm{C}$ before analysis.

Determination of the benzotriazoles' concentrations in the samples was performed according to $[9,45]$ with a detection limit of $0.01 \mu \mathrm{g} / \mathrm{L}$.

Blanks with the same values of the analytes were analyzed for possible contaminations of the experimental 
samples and for the accuracy of the method. The total error of the method amounted to $15 \%$.

\section{Fluorescence in situ hybridization}

For detecting changes in the ASC in the larger-scale setups 1 to 4, the ASC used as inoculum was initially analyzed (day 0 ) as well as the ASC from setups 1 to 4 and at the end of the experiment (day 22) by applying FISH. Table 3 shows the sequences and formamide concentrations of all used rRNA-targeted oligonucleotides. The probes (Eurofins MWG Operon, Ebersberg, Germany) were labeled at the $5^{\prime}$ end with the fluorescent dye indocarbocyanine (Cy3). All steps, i.e., cell fixation by paraformaldehyde and ethanol, staining with 4',6'diamidino-2-phenylindole (DAPI), the drying and washing procedure, were performed according to [46]. Microscopic analyses were performed with an Axioplan epifluorescence microscope (Carl Zeiss, Jena, Germany) to detect hybridized and DAPI stained cells. Positive hybridized cells were quantified using class indexes as described elsewhere [47], with category 0, denoting the lack of specific signals and category 5 the excessive occurrence thereof.

\section{Competing interests}

The authors declare that they have no competing interests.

\section{Authors' contributions}

$\mathrm{BaH}$ and $\mathrm{BeH}$ drafted the manuscript, designed, and performed the biodegradation experiments. HL reviewed the manuscript. $\mathrm{HH}$ and EM conceived of the study, participated in its coordination, and helped to review the manuscript. All authors read and approved the final manuscript.

\section{Acknowledgements}

Financial support by the Bavarian State Ministry of the Environment and Public Health (StMUG) is gratefully acknowledged.

\section{Author details}

${ }^{1}$ Urban Water Systems Engineering, Technische Universität München, Am Coulombwall, Garching 85748, Germany. ${ }^{2}$ Bavarian Environment Agency, Kaulbachstraße 37, Munich 80539, Germany. ${ }^{3}$ Karlsruhe Institute of Technology, Engler-Bunte-Institut, Bereich Wasserchemie und Wassertechnologie, Karlsruhe 76131, Germany.

Received: 14 August 2013 Accepted: 17 November 2013 Published: 25 November 2013

\section{References}

1. Verheyen V, Cruickshank A, Wild K, Heaven MW, McGee R, Watkins M, Nash D: Soluble, semivolatile phenol and nitrogen compounds in milk-processing wastewaters. J Dairy Sci 2009, 92:3484-3493.

2. Voutsa D, Hartmann P, Schaffner C, Giger W: Benzotriazoles, alkylphenols and bisphenol $A$ in municipal wastewaters and in the Glatt River, Switzerland. Environ Sci Pollut R 2006, 13:333-341.

3. Gruden $C L$, Dow SM, Hernandez MT: Fate and toxicity of aircraft deicing fluid additives through anaerobic digestion. Water Environ Res 2001, 73:72-79.

4. Janna H, Scrimshaw MD, Williams RJ, Churchley J, Sumpter JP: From dishwasher to tap? Xenobiotic substances benzotriazole and tolyltriazole in the environment. Environ Sci Technol 2011, 45:3858-3864.

5. Zhang Z, Ren N, Li Y-F, Kunisue T, Gao D, Kannan K: Determination of benzotriazole and benzophenone UV filters in sediment and sewage sludge. Environ Sci Technol 2011, 45:3909-3916.
6. Liu Y-S, Ying G-G, Shareef A, Kookana RS: Occurrence and removal of benzotriazoles and ultraviolet filters in a municipal wastewater treatment plant. Environ Pollut 2012, 165:225-232.

7. Hart DS, Davis LC, Erickson LE, Callender TM: Sorption and partitioning parameters of benzotriazole compounds. Microchem J 2004, 77:9-17.

8. Reemtsma T, Weiss S, Mueller J, Petrovic M, Gonzalez S, Barcelo D, Ventura F, Knepper TP: Polar pollutants entry into the water cycle by municipal wastewater: a European perspective. Environ Sci Technol 2006, 40:5451-5458.

9. Liu Y-S, Ying G-G, Shareef A, Kookana RS: Biodegradation of three selected benzotriazoles under aerobic and anaerobic conditions. Water Res 2011, 45:5005-5014.

10. Kiss A, Fries E: Seasonal source influence on river mass flows of benzotriazoles. J Environ Monit 2012, 14:697-703.

11. Kiss A, Fries E: Occurrence of benzotriazoles in the rivers Main, Hengstbach, and Hegbach (Germany). Environ Sci Pollut Res Int 2009, 16:702-710.

12. Nakata H, Murata S, Filatreau J: Occurrence and concentrations of benzotriazole UV stabilizers in marine organisms and sediments from the Ariake Sea, Japan. Environ Sci Technol 2009, 43:6920-6926.

13. Loos R, Locoro G, Comero S, Contini S, Schwesig D, Werres F, Balsaa P, Gans O, Weiss S, Blaha L, Bolchi M, Gawlik BM: Pan-European survey on the occurrence of selected polar organic persistent pollutants in ground water. Water Res 2010, 44:4115-4126.

14. Loos R, Gawlik BM, Locoro G, Rimaviciute E, Contini S, Bidoglio G: EU-wide survey of polar organic persistent pollutants in European river waters. Environ Pollut 2009, 157:561-568.

15. Wolschke H, Xie Z, Möller A, Sturm R, Ebinghaus R: Occurrence, distribution and fluxes of benzotriazoles along the German large river basins into the North Sea. Water Res 2011, 45:6259-6266.

16. Nakata H, Shinohara R-I, Nakazawa Y, Isobe T, Sudaryanto A, Subramanian A, Tanabe S, Zakaria MP, Zheng GJ, Lam PKS, Kim EY, Min BY, We SU, Viet PH, Tana TS, Prudente M, Frank D, Lauenstein G, Kannan K: Asia-Pacific mussel watch for emerging pollutants: distribution of synthetic musks and benzotriazole UV stabilizers in Asian and US coastal waters. Mar Pollut Bull 2012, 64:2211-2218.

17. Cancilla DA, Martinez J, Van Aggelen GC: Detection of aircraft deicing/ antiicing fluid additives in a perched water monitoring well at an international airport. Environ Sci Technol 1998, 32:3834-3835.

18. Seeland A, Oetken M, Kiss A, Fries E, Oehlmann J: Acute and chronic toxicity of benzotriazoles to aquatic organisms. Environ Sci Pollut R 2012, 19:1781-1790.

19. Kadar E, Dashfield S, Hutchinson TH: Developmental toxicity of benzotriazole in the protochordate Ciona intestinalis (Chordata, Ascidiae). Anal Bioanal Chem 2010, 396:641-647.

20. Pedrazzani R, Ceretti E, Zerbini I, Casale R, Gozio E, Bertanza G, Gelatti U, Donato F, Feretti D: Biodegradability, toxicity and mutagenicity of detergents: integrated experimental evaluations. Ecotoxicol Environ Saf 2012, 84:274-281.

21. Cancilla DA, Holtkamp A, Matassa L, Fang X: Isolation and characterization of Microtox ${ }^{\circledast}$-active components from aircraft de-icing/anti-icing fluids. Environ Toxicol Chem 1997, 16:430-434.

22. Cheng C, Phipps D, Alkhaddar RM: Treatment of spent metalworking fluids. Water Res 2005, 39:4051-4063.

23. Sahar E, Ernst M, Godehardt M, Hein A, Herr J, Kazner C, Melin T, Cikurel H, Aharoni A, Messalem R, Brenner A, Jekel M: Comparison of two treatments for the removal of selected organic micropollutants and bulk organic matter: conventional activated sludge followed by ultrafiltration versus membrane bioreactor. Water Sci Technol 2011, 63:733-740

24. Asimakopoulos AG, Ajibola A, Kannan K, Thomaidis NS: Occurrence and removal efficiencies of benzotriazoles and benzothiazoles in a wastewater treatment plant in Greece. Sci Total Environ 2013, 452-453:163-171.

25. Stasinakis AS, Thomaidis NS, Arvaniti OS, Asimakopoulos AG, Samaras VG, Ajibola A, Mamais D, Lekkas TD: Contribution of primary and secondary treatment on the removal of benzothiazoles, benzotriazoles, endocrine disruptors, pharmaceuticals and perfluorinated compounds in a sewage treatment plant. Sci Total Environ 2013, 463-464:1067-1075.

26. Weiss S, Jakobs J, Reemtsma T: Discharge of three benzotriazole corrosion inhibitors with municipal wastewater and improvements by membrane bioreactor treatment and ozonation. Environ Sci Technol 2006, 40:7193-7199.

27. Reemtsma T, Miehe U, Duennbier U, Jekel M: Polar pollutants in municipal wastewater and the water cycle: occurrence and removal of benzotriazoles. Water Res 2010, 44:596-604.

28. Hollender J, Zimmermann SG, Koepke S, Krauss M, McArdell CS, Ort C, Singer H, von Gunten U, Siegrist H: Elimination of organic micropollutants in a municipal wastewater treatment plant upgraded with a full-scale postozonation followed by sand filtration. Environ Sci Technol 2009, 43:7862-7869. 
29. Weiss $S$, Reemtsma T: Membrane bioreactors for municipal wastewater treatment - a viable option to reduce the amount of polar pollutants discharged into surface waters? Water Res 2008, 42:3837-3847.

30. Matamoros V, Jover E, Bayona JM: Occurrence and fate of benzothiazoles and benzotriazoles in constructed wetlands. Water Sci Technol 2010, 61:191-198.

31. Winkler CK, Tasnádi G, Clay D, Hall M, Faber K: Asymmetric bioreduction of activated alkenes to industrially relevant optically active compounds. $J$ Biotechnol 2012, 162:381-389.

32. Hall M, Stueckler C, Hauer B, Stuermer R, Friedrich T, Breuer M, Kroutil W, Faber K: Asymmetric bioreduction of activated $\mathrm{C}=\mathrm{C}$ bonds using zymomonas mobilis NCR enoate reductase and old yellow enzymes OYE 1-3 from yeasts. European J Org Chem 2008, 2008:1511-1516.

33. Stueckler C, Hall M, Ehammer H, Pointner E, Kroutil W, Macheroux P, Faber K: Stereocomplementary bioreduction of $\alpha$, $\beta$-unsaturated dicarboxylic acids and dimethyl esters using enoate reductases: enzyme- and substrate-based stereocontrol. Org Lett 2007, 9:5409-5411.

34. Tauber K, Hall M, Kroutil W, Fabian WMF, Faber K, Glueck SM: A highly efficient ADH-coupled NADH-recycling system for the asymmetric bioreduction of carbon-carbon double bonds using enoate reductases. Biotechnol Bioeng 2011, 108:1462-1467.

35. Manz W, Wagner M, Amann R, Schleifer K-H: In situ characterization of the microbial consortia active in two wastewater treatment plants. Water Res 1994, 28:1715-1723.

36. Snaidr J, Amann R, Huber I, Ludwig W, Schleifer KH: Phylogenetic analysis and in situ identification of bacteria in activated sludge. Appl Environ Microbiol 1997, 63:2884-2896.

37. Björnsson L, Hugenholtz P, Tyson GW, Blackall LL: Filamentous Chloroflexi (green non-sulfur bacteria) are abundant in wastewater treatment processes with biological nutrient removal. Microbiology 2002, 148:2309-2318.

38. Manz W, Amann R, Ludwig W, Wagner M, Schleifer K-H: Phylogenetic oligodeoxynucleotide probes for the major subclasses of proteobacteria: problems and solutions. Syst Appl Microbiol 1992, 15:593-600.

39. Neef A: Anwendung der in situ-Einzelzell-Identifizierung von Bakterien zur Populationsanalyse in komplexen mikrobiellen Biozönosen. In Technische Universität München. Munich; 1997.

40. Manz W, Amann R, Ludwig W, Vancanneyt M, Schleifer K-H: Application of a suite of $16 \mathrm{~S}$ rRNA-specific oligonucleotide probes designed to investigate bacteria of the phylum cytophaga-flavobacter-bacteroides in the natural environment. Microbiology 1996, 142:1097-1106.

41. Meier H, Amann R, Ludwig W, Schleifer KH: Specific oligonucleotide probes for in situ detection of a major group of gram-positive bacteria with low DNA G + C content. Syst App/ Microbiol 1999, 22:186-196.

42. Erhart R: In situ Analyse mikrobieller Biozönosen in Abwasserreinigungsanlagen. Munich: Technische Universität München; 1997.

43. Wagner $M$, Amann $R$, Lemmer $H$, Schleifer KH: Probing activated sludge with oligonucleotides specific for proteobacteria: inadequacy of culturedependent methods for describing microbial community structure. Appl Environ Microbiol 1993, 59:1520-1525.

44. Schmid M, Thill A, Purkhold U, Walcher M, Bottero JY, Ginestet P, Nielsen PH, Wuertz S, Wagner M: Characterization of activated sludge flocs by confocal laser scanning microscopy and image analysis. Water Res 2003, 37:2043-2052.

45. Herzog B, Lemmer H, Huber B, Horn H, Müller E: Xenobiotic benzotriazoles-biodegradation under meso- and oligotrophic conditions as well as denitrifying, sulfate-reducing, and anaerobic conditions. Environ Sci Pollut R 2013:1-10

46. Amann RI: In situ identification of micro-organisms by whole cell hybridization with rRNA-targeted nucleic acid probes. In Molecular Microbial Ecology Manual. Edited by Akkermans AL, Elsas J, Bruijn F. Netherlands: Springer; 1995:331-345.

47. Muller E, Schade M, Lemmer H: Filamentous scum bacteria in activated sludge plants: detection and identification quality by conventional activated sludge microscopy versus fluorescence in situ hybridization. Water Environ Res 2007, 79:2274-2286.

\section{doi:10.1186/2190-4715-25-31}

Cite this article as: Herzog et al:: Analysis and in situ characterization of activated sludge communities capable of benzotriazole biodegradation. Environmental Sciences Europe 2013 25:31. 